\title{
UDINE RADIOCARBON LABORATORY DATE LIST II
}

\author{
VALERIO BARBINA, FRANCO CALLIGARIS, ADRIANO DEL \\ FABBRO, and ALESSANDRO TURELLO \\ Centro di Ricerca Applicata e Documentazione \\ Viale Leonardo da Vinci 16, 33100 Udine, Italy
}

\section{INTRODUCTION}

This list includes the measurements carried out from 1977-1978. Procedures of synthesis of benzene, counting method, and performance figures of results were previously described (R, 1982, v 24, p 214-216; CRAD, 1977; Barbina, Calligaris, \& Ciuti, 1979). Samples were pretreated according to generally applied methods, depending on type of material and particular archaeologic and geologic features.

\section{SAMPLE DESCRIPTIONS \\ CROSS-CHECK SAMPLES}

The following samples were dated in order to check the reliability of laboratory procedures and measurements, by comparison with the results obtained on the same samples by other laboratories, and with archaeologic dating.

\section{UD-16. Mezzano II, 35 D}

Charcoal from Late Bronze age layers in Mezzano little crater lake in caldera of Latera, Vulsini Mts, volcanic region, Comm Valentano, prov Viterbo, Latium, Italy (46 $\left.36^{\prime} 30^{\prime \prime} \mathrm{N}, 11^{\circ} 46^{\prime} 08^{\prime \prime} \mathrm{E}\right)$. Subm 1976 by G Belluomini, Radiocarbon Dating Lab, Univ Rome. Comment: date perfectly agrees with results obtained in Rome, R-994B:2970 \pm 60 .

\section{Sahara series}

UD-17.

$7630 \pm 250$

Charcoal from Neolithic layers of Wadi Teshuinat, Acacus, Fezzan, Libya $\left(25^{\circ} 30^{\prime} 14^{\prime \prime} \mathrm{N}, 11^{\circ} 45^{\prime} 38^{\prime \prime} \mathrm{E}\right)$. Subm 1977 by G Belluomini. Comment: date agrees with results obtained in Rome, R-1033:7900 \pm 300 .

UD-73. Deir el Medina-Luxor

$3680 \pm 110$

Fragment of cloth from tomb of Kha XVIII Dynasty, Deir el Medina, W side Thebe, Luxor, Egypt (25 $48^{\prime} 24^{\prime \prime}$ N, $32^{\circ} 28^{\prime} 19^{\prime \prime}$ E). Subm 1977 by $\mathrm{S}$ Curto, Egyptian Mus, Torino, Italy. Comment: date agrees with archaeol estimated age: $3940 \pm 20$.

\section{ARCHAEOLOGIC SAMPLES}

Coal and ash samples

UD-1. Wadi el Bouzna

Coal and ashes from clayey layers of Wadi el Bouzna, Fezzan, Libya $\left(26^{\circ} 24^{\prime} 29^{\prime \prime} \mathrm{N}, 14^{\circ} 21^{\prime} 15^{\prime \prime} \mathrm{E}\right)$. Coll 1971 by A Del Fabbro (1970) and V Barbina (Reygasse, 1934). Banks of Wadi el Bouzna have yielded many flint implements, such as biconvex axes, broad cutters, and chisels. Lithic 
industry typology suggests likeness between Gargano and Teneré stations and Wadi el Bouzna, whereas pottery typology shows remarkable differences from Campignian culture. Comment: ${ }^{14} \mathrm{C}$ date agrees with expected age range inferred by ${ }^{14} \mathrm{C}$ measurements of Teneré layers (Alimen, Beucher, \& Conrad, 1966; Beucher, 1971).

\section{Pozzuolo series}

Charcoal from cremation tombs of Pozzuolo necropolis, Italy $\left(45^{\circ} 50^{\prime}\right.$ $18^{\prime \prime} \mathrm{N}, 13^{\circ} 10^{\prime} 39^{\prime \prime} \mathrm{E}$ ) (Candussio, 1980; Cassola, 1980). Graves were dug in silty-clayey soil, and dated by assoc archaeol material, such as pottery and bronze objects. ${ }^{14} \mathrm{C}$ dates agree with archaeol estimated age and TL pottery dating.

UD-57. Tombe No. 1

$2250 \pm 100$

Charcoal from cremation tomb No. 1 of Pozzuolo necropolis. Coll and subm 1977 by A Del Fabbro and A Candussio, Dept Antiquities, Udine, Italy.

UD-58. Tombe No. 2

$2700 \pm 100$

Charcoal from cremation tomb No. 2 of Pozzuolo necropolis. Coll and subm 1977 by A Del Fabbro and A Candussio.

\section{Wood samples}

UD-33. Tempietto Longobardo-Cividale del Friuli $\quad \mathbf{3 4 0} \pm \mathbf{3 0}$

Wood from beam found above plaster walls of Lombard chapel, "Tempietto Longobardo", Cividale del Friuli, Italy (Civiletti, 1960) $\left(46^{\circ}\right.$ $05^{\prime} 30^{\prime \prime} \mathrm{N}, 13^{\circ} 25^{\prime} 50^{\prime \prime} \mathrm{E}$ ) during restoration in 1977 after 1976 earthquake. Coll by A Del Fabbro and V Barbina and subm by G Del Basso, Municipality Cividale del Friuli. Comment: recent age of sample suggests restoration was undertaken ca AD 1520, due to 1511 earthquake.

\section{Wood samples}

\section{GEOLOGIC SAMPLES}

\section{UD-41. Palazzo delle Albere-Trento}

$9800 \pm 200$

Sample found during geognostic drillings in Palazzo delle Albere area, Trento, Italy $\left(46^{\circ} 12^{\prime} 31^{\prime \prime} \mathrm{N}, 11^{\circ} 07^{\prime} 14^{\prime \prime} \mathrm{E}\right)$ in gray-blue silt of Adige $\mathrm{R}$ at depth $10 \mathrm{~m}$. Sand and gravel layer, deposited by Fersina stream, overlays these sediments. Coll and subm 1977 by Tridentine Mus Nat Hist, Trento, Italy. Comment: ${ }^{14} \mathrm{C}$ dating allowed assessment of rate of alluviation of Adige valley, and period during which Fersina $\mathrm{R}$ sediments reached this area.

\section{UD.42. Tovel Lake}

$610 \pm 60$

Fragment from uprooted tree trunk at depth $15 \mathrm{~m}$ in Tovel lake, Dolomiti di Brenta, Trento, Italy $\left(46^{\circ} 15^{\prime} 30^{\prime \prime} \mathrm{N}, 10^{\circ} 57^{\prime} 12^{\prime \prime} \mathrm{E}\right)$. Coll by Sub Club "Trento" and subm 1977 by G Tomasi, Tridentine Mus Nat Hist. Comment: ${ }^{14} \mathrm{C}$ age suggests lake level was lower than at present, correlated with cold climate of alpine region during that period. 
Peat and mollusk shell samples

Results reported here are part of study of paleography of lagoon of Venice made by Ist Studio della Dinamica delle Grandi Masse CNR, Venice, Italy, as previously described (R, 1982, v 24, p 214-216; Gatto, 1980).

Laguna di Venezia

\section{Ca' Bianca series}

Peat from lagoon of Venice, Ca' Bianca $\left(45^{\circ} 23^{\prime} 33^{\prime \prime} \mathrm{N}, 12^{\circ} 21^{\prime} 18^{\prime \prime}\right.$ E). Coll by $P$ Da Roit, Lab Geol Appl CNR Padova, and subm by $\mathbf{P}$ Gatto, Ist Studio Dinamica Grandi Masse, CNR, Venice.

UD-10.

$24,100 \pm 1500$

Peat from drilling 8 at depth $28.1 \mathrm{~m}$.

\section{Lido series}

Peat and shells from lagoon of Venice, Lido $\left(45^{\circ} 23^{\prime} 53^{\prime \prime} \mathrm{N}, 12^{\circ} 19^{\prime}\right.$ 03" E). Coll 1974 by P Da Roit and subm by P Gatto.

UD-43.

$4820 \pm 150$

Carbonate (mollusk shells) from drilling 11 at depth $10.3 \mathrm{~m}$.

UD-44.

$21,800 \pm 300$

Peat from drilling 11 at depth $22.55 \mathrm{~m}$.

\section{S Andrea series}

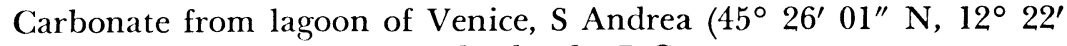
53" E). Coll 1974 by P Da Roit and subm by P Gatto.

UD-45.

$5970 \pm 100$

Carbonate (mollusk shells) from drilling 13 at depth $8.4 \mathrm{~m}$.

\section{Cavallino Punta Sabbioni series}

Peat from lagoon of Venice, Cavallino Punta Sabbioni (45 25' 58" $\left.\mathrm{N}, 12^{\circ} 22^{\prime} 10^{\prime \prime} \mathrm{E}\right)$. Coll 1974 by P Da Roit and subm by P Gatto.

\section{UD-54.}

$24,500 \pm 1000$

Silty peat from drilling at depth $28.52 \mathrm{~m}$.

\section{Sottomarina Lido series}

Peat from lagoon of Venice, Sottomarina Lido $\left(45^{\circ} 15^{\prime} 49^{\prime \prime} \mathrm{N}, 12^{\circ} 17^{\prime}\right.$ $38^{\prime \prime}$ E). Coll 1974 by P Da Roit and subm by P Gatto.

UD-60.

$$
1800 \pm 80
$$

Peat from drilling 1 at depth $8.8 \mathrm{~m}$.

\section{Sottomarina series}

Peat from lagoon of Venice, Sottomarina $\left(45^{\circ} 16^{\prime} 34^{\prime \prime} \mathrm{N}, 12^{\circ} 18^{\prime} 39^{\prime \prime}\right.$

E). Coll 1974 by P Da Roit and subm by P Gatto.

UD-63.

$10,630 \pm 100$

Sandy peat from drilling 2 at depth $22.6 \mathrm{~m}$. 


\section{Cavallino Ca' Ballerin series}

Peat and carbonate (mollusk shells) from lagoon of Venice, Cavallino Ca' Ballerin $\left(45^{\circ} 30^{\prime} 18^{\prime \prime} \mathrm{N}, 12^{\circ} 25^{\prime} 21^{\prime \prime} \mathrm{E}\right)$. Coll 1974 by P Da Roit and subm by P Gatto.

UD-64.

$4670 \pm 150$

Peat from drilling at depth $12.75 \mathrm{~m}$.

UD-64.

$\mathbf{2 0 , 5 0 0} \pm \mathbf{8 0 0}$

Carbonate from drilling 18 at depth $16.55 \mathrm{~m}$.

\section{Cavallino Ca' Pasquali series}

Peat from lagoon of Venice, Cavallino Ca' Pasquali $\left(45^{\circ} 28^{\prime} 21^{\prime \prime} \mathrm{N}\right.$, $12^{\circ} 33^{\prime} 48^{\prime \prime}$ E). Coll 1974 by P Da Roit and subm by P Gatto.

UD.68.

$\mathbf{5 4 1 0} \pm \mathbf{7 0}$

Peat from drilling 18 at depth $10.5 \mathrm{~m}$.

\section{REFERENCES}

Alimen, H, Beucher, F, and Conrad, G, 1966, Chronologie du dernier cycle pluvial aride au Sahara nord-occidental: Mem Acad Sci, Paris, v 263, p 5-15.

Barbina, V, Calligaris, F, and Ciuti, P, 1979, An apparatus for beta-counting used in a radiocarbon dating laboratory: Nuclear Instruments and Methods, v 163, p 545-548.

Beucher, F, 1971, Etude palinologique des formations néogènes et quaternaires au Sahara occidental: Centre Documents CNRS No. 5408, Paris, v 3, p 187-196.

Candussio, A, 1980, Recenti scoperte preistoriche a Pozzuolo del Friuli: Udine, Soc Filologica Friulana, p 78-84.

Cassola, P, 1980, Ricerche a Pozzuolo del Friuli: Trieste, Mem Civici Mus Storia ed Arte, v 2, p 148-165.

Civiletti, B, 1960, Ricerche nuove sul tempietto longobardo di Cividale del Friuli: Venice, Arte veneta, p 18-21.

CRAD, 1977, Servizio di radiodatazione con ${ }^{14} \mathrm{C}$ : Udine, Bull no. 1.

Del Fabbro, A, 1970, Giacimento ed industria campignana nei pressi di Wadi el Bouzna, Fezzan, Libya: Udine, Mondo sotterraneo, p 18-37.

Gatto, P, 1980, Il sottosuolo del litorale veneziano: Tech rept No. 108, Venice, Ist Studio Dinamica Grandi Masse, CNR, p 1-18.

Reygasse, M, 1934, Découverte d'une technique campignienne dans le paléolitique inferieur de Sud Costantinois: Mem préhist de France, Cong, Ilth, p 570-573. 